\section{La odontología digital en rehabilitación oral}

\section{Digital dentistry in oral rehabilitation}

Eduardo Piza Pellizzer 1,a, Jessica Marcela de Luna Gomes 1,b

${ }^{1}$ Universidade Estadual Paulista "Júlio de Mesquita Filho", Faculdade de Odontologia de Araçatuba, (FOA-UNESP) Araçatuba, São Paulo, Brasil.

a Doctor en Odontología, área de concentración: Materiales dentarios.

${ }^{\text {b } M a g i s t e r ~ e n ~ O d o n t o l o g i ́ a, ~ a ́ r e a ~ d e ~ c o n c e n t r a c i o ́ n: ~ P r o ́ t e s i s ~ d e n t a r i a . ~}$

\section{Correspondencia:}

Eduardo Piza Pellizzer: ed.pl@uol.com.br

Universidade Estadual Paulista "Júlio de Mesquita Filho", Faculdade de Odontologia de Araçatuba. Rua José Bonifácio 1193, Vila Mendonça, Araçatuba, São Paulo, Brasil. CEP:16015-050.

ORCID: 0000-0003-0670-5004

\section{Coautora:}

Jessica Marcela de Luna Gomes: jessicamgomes@hotmail.com ORCID: 0000-0002-2621-6200

Recibido: 24/01/21

Aceptado: 29/01/21

Publicado: 01/04/21
El auge de la odontología digital simplificó la confección de infraestructuras, y permitió el uso de diferentes materiales (cerámicas, metal, cera, entre otros), confeccionando prótesis en menos tiempo y asegurando la precisión de la interfaz entre la corona y la preparación dental o entre el implante y la prótesis ${ }^{1,2}$. Los sistemas CAD-CAM (Computer-aided Design and Computer-aided Manufacturing) se utilizan en rehabilitaciones extensas (prótesis híbridas, sobredentaduras, prótesis totales y removibles), parciales (prótesis fijas sobre dientes o implantes) y prótesis unitarias, así como auxiliar en la planificación de la rehabilitación y cirugía, placas oclusales, confección de modelos, entre otros. Los sistemas $C A D-C A M$ se pueden subdividir en: intra y extraorales (escáner de mesa), o también con relación a la disponibilidad en la manipulación del archivo digital (stl.): sistemas cerrados (escaneo y fresado de la misma empresa) y sistemas abiertos (escaneo y fresado/impresión independiente).

La tendencia de los laboratorios de prótesis es adquirir sistemas extraorales abiertos, con la finalidad de facilitar la demanda de trabajo, ya que los sistemas extraorales presentan un flujo de trabajo mixto entre lo digital y lo convencional. El clínico envía el molde o el modelo al laboratorio y este es el responsable de todas las demás etapas de confección. El sistema extraoral presenta cierta precisión ${ }^{3}$, entretanto presenta un mayor número de etapas que son susceptibles a una correcta manipulación de los materiales de impresión, del material de revestimiento y la deformación de las aleaciones metálicas. Por tanto, es fundamental que el especialista y el laboratorio de prótesis cumplan estrictamente con todas las etapas para reducir las posibilidades de error.

Actualmente, los sistemas intraorales han ido ganando espacio en las clínicas, sin embargo, debido al alto costo en la adquisición de los aparatos, las empresas de tecnología están apostando por el desarrollo de dispositivos más pequeños y a costo reducido, con el fin de ganar más espacio en el mercado. Los sistemas intraorales tienen un alto grado de satisfacción del paciente y un tiempo clínico más corto. Los estudios ${ }^{4,5}$ indican un bajo costo y precisión en la adaptación de las rehabilitaciones. El digital workflow permite que el paciente complete su tratamiento en una o dos sesiones clínicas. A la vanguardia del digital workflow, surgieron las impresoras 3D, una alternativa a las fresadoras. Las impresoras tienen la capacidad de confeccionar en poco tiempo: prótesis provisionales, placas oclusales y más recientemente bases para dentaduras totales, con menos tiempo de trabajo y costo.

Investigaciones recientes ${ }^{6,7}$ indican que la eficacia en la precisión de los sistemas puede estar relacionada con el tipo de rehabilitación. Por ejemplo, los sistemas $C A D$ $C A M$, principalmente los intraorales, deben usarse con precaución en las rehabilitaciones extensas, porque el 
escáner intraoral aún no es capaz de capturar imágenes con precisión de un reborde desdentado o incluso la ubicación/posición exacta de los implantes en rehabilitaciones con implantes dentales del tipo prótesis híbrida o sobredentadura.

La innovación de los sistemas $C A D-C A M$ también está relacionada con la aparición de nuevos materiales dentales específicos para estos sistemas y que son fundamentales en la longevidad de las rehabilitaciones. Se observa en la literatura que el uso de sistemas está directamente asociado a un aumento en el número de restauraciones totalmente cerámicas, principalmente del tipo monolíticas (zirconia, disilicato de litio, nanocerámicas, cerámicas híbridas), ya sea en rehabilitación sobre implantes o dientes, aumentando el grado estético. Además, cabe mencionar que, en las prótesis fijas de tres o más elementos o en las barras de prótesis híbridas, se prefiere el fresado en aleaciones metálicas o en cera para su posterior sobrefundición, porque estos materiales están sujetos a la técnica de soldadura por puntos, a diferencia de las cerámicas. La técnica del punto de soldadura ayuda a obtener una mejor pasividad y adaptación, compensando posibles errores de escaneo o alguna etapa de confección, como se mencionó anteriormente.

Hace 10 años iniciamos en nuestro grupo de investigación en la Facultad de Odontología de Araçatuba, UNESP (São Paulo, Brasil), una línea de investigación sobre tecnología digital aplicada a la Odontología, con el fin de verificar la precisión de los sistemas digitales que estaban surgiendo, en la fabricación de prótesis fijas sobre dientes o implanto-soportadas. Desde entonces, hemos tenido la oportunidad de trabajar con diferentes escáneres, materiales y laboratorios y estos tres pilares son inseparables en la construcción de proyectos de rehabilitación que presentan precisión y longevidad, con resultados prometedores. Además, decidimos incluir la realidad de la tecnología digital en la docencia de pregrado, con una disciplina en la que los estudiantes puedan tener contacto con este universo.

En las diversas áreas odontológicas, la inserción de la tecnología digital fue una innovación en la forma de comunicación entre paciente, clínico y laboratorios con una atención cada vez más individualizada con alta precisión y estética. Se cree que el futuro es el perfeccionamiento y la creación de materiales específicos para tecnología digital y la creación de sistemas por parte de empresas y universidades que combinen precisión y bajo costo. Aportamos en este editorial una visión realista y actual de la aplicabilidad clínica de los sistemas CAD-CAM. Tal vez, en un tiempo el escenario cubierto en este editorial haya cambiado y la odontología digital sea más que una realidad, una práctica diaria en la clínica dental, para todo tipo de rehabilitación presentando precisión y longevidad. El futuro es promisorio.

\section{Referencias bibliográficas}

1. Pellizzer EP, Kimpara ET, Miyashita E. Prótese Sobre Implante - Baseado em Evidências Cientificas. 1a ed. São Paulo: Editora Napoleão; 2016.

2. Perri E, Pellizzer EP. Fundamentos em Implantodontia - Uma Visão Contemporânea. 2a ed. Rio de Janeiro: Editora Santos; 2015.

3. Mello CC, Santiago Junior JF, Lemos CAA, Galhano GA, Evangelisti E, Scotti R, et al. Evaluation of the accuracy and stress distribution of 3-unit implant supported prostheses obtained by different manufacturing methods. Mater Sci Eng C Mater Biol Appl. 2019;102:66-74. DOI: $10.1016 /$ j.msec.2019.03.059.

4. de Oliveira NRC, Pigozzo MN, Sesma N, Laganá DC. Clinical efficiency and patient preference of digital and conventional workflow for single implant crowns using immediate and regular digital impression: A meta-analysis. Clin Oral Implants Res. 2020;31(8):669-86. DOI: 10.1111/clr.13604.

5. Mangano F, Veronesi G. Digital versus Analog Procedures for the Prosthetic Restoration of Single Implants: A Randomized Controlled Trial with 1 Year of Follow-Up. Biomed Res Int. 2018;2018:5325032. DOI: $10.1155 / 2018 / 5325032$.

6. Kalberer N, Mehl A, Schimmel M, Müller F, Srinivasan $\mathrm{M}$. CAD-CAM milled versus rapidly prototyped (3D-printed) complete dentures: An in vitro evaluation of trueness. J Prosthet Dent. 2019;121(4):637-43. DOI: 10.1016/j.prosdent.2018.09.001.

7. Dutton E, Ludlow M, Mennito A, Kelly A, Evans Z, Culp A, et al. The effect different substrates have on the trueness and precision of eight different intraoral scanners. J Esthet Restor Dent. 2020;32(2):204-18. DOI: $10.1111 /$ jerd. 12528 . 